\title{
A MATHEMATICAL MODELING OF CLIMATE CHANGING VIA EL NIÑO AND LA NIÑA EFFECTS
}

DOI: http://dx.doi.org/10.18509/GBP.2017.06

UDC: 551.583.16:519.87

\section{Bhupendra Nath Tiwari \\ S. Chandra Kishore Ninoslav Marina}

University of Information Science \& Technology "St. Paul the Apostle" - Ohrid, Partizanska Str. bb 6000 Ohrid, Macedonia

\begin{abstract}
Climate changing is one of the greatest threats towards the survival of mankind on the earth. This motivates us to investigate climatic changing effects via fluctuations of the temperature, wind patterns and precipitation taking place in a given geoenvironment. Following the fact that a minor variation in the average temperature of the earth can render potentially harmful changes both in the climate and weather, we offer temperature based modeling of the global warming over a given time duration. This provides an interpolating atmospheric temperature as a function of the wind velocity and humidity. We further estimate the modeling error in prediction of the temperature over infinitesimal variations of the origin in the space of wind patterns and precipitation. Our study is well-suited for effective managements of natural hazards, natural resources and geographic information system planning.
\end{abstract}

Keywords: Climatology, El Niño \& La Niña, SOI, Geo-environment, GIS

\section{INTRODUCTION}

Climatic changes emerge as an alternation caused by various climate effects which are maintained over a certain period of time. Such variations could be classified on the basis of changes in temperature, wind velocity and humidity which take place over a given geobasis [1]. Global warming is commonly referred as the main reason for the climatic change. Global warming can be explained as the change in average temperature over the earth surface. Namely, the aforementioned changes in atmospheric temperature occur largely due to an increase in the concentration of various greenhouse gases, such as methane, carbon dioxide, ozone, nitrous oxide and water vapor [2]. In the past century, the average temperature of earth is reported to have risen by $1.5^{\circ} \mathrm{F}$. It is equally predicated that in the next hundred years it could even rise to $8.6^{\circ} \mathrm{F}$, see [2]. This increment in the average temperature of earth is a serious threat to the climate. As per the above mentioned facts [2], it is noteworthy to realize that there are natural occurrences of disasters including floods, droughts and heat waves which mostly happen because of abnormal rainfalls. In addition, there are also serious effects concerning the rise of water levels of oceans and melting of glaciers, as well. It is therefore understood that there would likewise be continued rising in the sea level, warming of water in ocean, presence of acidity in water, melting of ice caps, etc.. Amongst the aforementioned phenomena, it is 
worth emphasizing that these factors largely contribute in changing the climate which definitely poses a challenge question to us in order to protect our ecological system [2], wherefore the survival of mankind on earth.

By only analyzing, it is well understood that human beings hold the main responsibility behind these climate changing issues. Indeed, there are other responsible factors which undermine an augmentation in the level of greenhouse gases in our atmosphere [2]. These mainly take place due to deforestation, burning of fossil fuels, industrial process and agricultural methods that generate emission of gases to atmosphere. The above activities of humans lead to the greenhouse effect [3]. The greenhouse gases act like a blanket over the earth surface. This cause the atmosphere to get warm by trapping the energy and make the essential feature for the survival of life on earth. Following an excessive emission of gases to the atmosphere, there exist observations showing various holes in blanket layer [3]. This paves path for the UV rays emerging from the sun to directly fall on the earth, which eventually leads to the global warming and diverse phenomena. As the result, atmospheric changes can definitely affect our physical geography including the health, agriculture, natural resources and water supply. It is worth mentioning that high emissions of the carbon dioxide, carbon monoxide and other harmful gases are largely responsible in changing the climate of the earth. Indeed, the carbon dioxide has an ability to exist in the atmosphere for more than a century, see [3] for an instance. An apt method to avoid such ecological changes is to exercise a reduced emission of the greenhouse gases.

Hereby, with a particular emphasis on global warming, we focus on El Niño and La Niña effects which are events of the natural global climate system. Namely, they occur naturally when the Pacific Ocean and the atmosphere above it changes from the normal states. This happens because of several reasons. The major reason responsible for the El Niño and La Niña effects arise via changes in the temperature profiles in the atmosphere and Equatorial Pacific Ocean [4]. Because of these variations in temperature, the effects of El Niño and La Niña are widely spread both in human beings and environment. From the perspective of the El Niño effects, it is worth mentioning that these are the events that are associated with an increasing warming of the central and eastern pacific. As a matter of the fact, the acronym El Niño signifies the little boy or the Christ child in Spanish which occurs around the December period of the year. Technically, the term El Niño refers to the large scale ocean atmosphere climate interactions linked to a periodic warming in sea surface temperatures across the central and east central Equatorial Pacific. On the other hand, the La Niña episodes represent periods of below average sea surface temperature across the east central Equatorial Pacific. This period is sometime called the little girl in Spanish in contrast to the El Niño episodes. Concerning the same, Ref. [4] offers a prior modeling of climate changing and greenhouse effects.

El Niño and La Niña effects are the opposite phases of what is jointly known as the El Niño southern oscillation (ENSO) cycles [4]. In this concern, El Niño is sometimes referred to the warm phase of the ENSO cycle, whilst the La Niña as it's cold phase. It is worth emphasizing the question: what causes terming the ENSO cycles as the El Niño and La Niña periods? The reason behind it follows from the fact that the above ENSO cycles are called the El Niño for the warm phase and La Niña for the other because of their Spanish acronyms. As mentioned before, the term El Niño in Spanish signifying the 'boy-child' which originally appeared around the Christmas corresponds to a warm ocean current in the southern coast. It is now used to describe the warming of the central and eastern tropical Pacific Ocean, while the La Niña is sometimes referred to as the cold 
phase of the aforementioned ENSO cycle. From the perspective of the El Niño effects, we find that they typically occur almost every five years and in due course what happens is that the warming in the ocean as caused by winds leads to diffusion of this warming as the temperature waves all over the globe. It non-linearly changes the atmospheric temperature, resulting in rainfall, wind patterns and variations in sea surface temperatures. Such changes could be both advantageous and harmful to the human beings and geoenvironment. For example, El Niño weakens an instance of hurricanes in the Atlantic and greenhouse effect based ecological warming, whereby it governs the atmospheric changes in Europe [4].

On the other hand, La Niña equally affects the atmospheric pressure, temperature, rainfall and the ocean temperature. In Europe, as mentioned above that the El Niño effects reduce the chances of autumnal hurricanes. This lead to a milder winter in the Northern part of Europe, especially in the United Kingdom, however one finds a colder winter in its other parts, viz. the western and southern Europe. Consequently, the above El Niño effects lead to snow falls in Mediterranean regions. In this paper, we anticipate that a wilder La Niña effect enables us in modelling the following environmental changing predictions: (i) stronger winds along the equatorial region, especially in the Pacific, (ii) decreased conviction in the Pacific leading to a weaker jet stream, (iii) temperatures are above the average in the southeast and below average in the northwest, (iv) conditions are more favorable for hurricanes in the Caribbean and central Atlantic area, and (v) greater instances of tornadoes in those states of US which are already vulnerable to them, see [4] for a review. An investigation concerning the above effects is modelled for infinitesimal variations in this paper. The corresponding chaotic cases are left open for a perspective research.

The rest of the paper is organized as follows. In Section 2, we offer a basic review of atmospheric phenomena concerning the global warming. In Section 3, we model the temperature as a function of the wind velocity and humidity of the atmosphere with associated analysis of the truncation error along with its implications via the southern oscillation index (SOI) fine tuning. In Section 4, we conclude the paper with remarks for future research developments towards the forecasting of ENSO states.

\section{REVIEW OF CLIMATOLOGY}

In this section, we offer basics of atmospheric phenomena undermining the global warming of the earth. As far as the earth science investigations are concerned, the climate changes can largely be linked with the branch of physical geography related to the study of the planet earth. Hereby, the climatology falls amongst important topics in the realism of geoscience and earth science studies. As a matter of the fact, the climatology affects both the interior and exterior parts of the earth including atmosphere, biosphere, hydrosphere and others. On the other hand, it follows that physical geography possesses drastic effects in exploring various patterns and processes undermining the human geography [5]. This follows from the fact that the core objective of the physical geography is to analyze the natural phenomena related to the exterior part of the earth, see [5] for a detailed treatment.

Hereby, El Niño and La Niña based temperature modulations impede the study of climatology [4], which emerge under fluctuating wind velocity and humidity. Indeed, an apt forecasting of the temperature plays an important role in climatology in the light of the present study of climate changing. Notice that the climatology emerges as a branch of science, which is designed to study atmospheric conditions and associated processes. This 
in particular includes oceanography and biogeochemistry. In this paper, we discuss the modelling of oceanography and leave open the implications concerning biogeochemistry for a separate research. Indeed, an apt whether forecasting is achieved via basic knowledge in climate with reference to EI Niño-southern oscillation, North Atlantic oscillation, Madden-Julian oscillation and Arctic oscillation, see [6] for an evaluation of the global atmospheric and land modelling simulations. On the other hand, in the light of biogeography, we may view this fact that the planet earth is covered with various species of ecosystems. Hereby, the branch of physical geography that includes the study of both animals and plants in a geographic space commonly concentrates in exploring the facets of the biogeography.

Furthermore, the geoenvironment studies support in exploring various atmospheric phenomena. Namely, an apt exploration of the environment, earth surface and its subsurfaces could be examined under the unified category of geoenvironment, see [7] for policy makings and their practices. This correspondingly deals with the relation of geology to geosynthetic materials. An investigation of these types in particular includes the effects of pollutants on earthen materials. Such pollutants amongst the others constitute natural hazards in the environment, whereby various threats, which can create negative impact on both the mankind and environment. Such phenomena are collectively called as natural hazards. In the light of hurricane vulnerability and Caribbean based damages in Latin America, the potentially natural causes including cyclonic storms, flood, drought and earthquakes are further categorized in terms of geophysical hazards. On the same footing, it follows that different diseases and plague fall under a unified agenda of the biological hazards.

Our study does not stop here, but it continues in other domains, as well. In the light of natural resources, it is worth mentioning that different resources that are formed without any contribution of human beings can be termed as natural resources. Such studies constitute a refined examination of various minerals, lands, water sources, atmosphere phenomena, sunlight and other ecological occurrences. This also encompasses the effect of local electric and magnetic fields present in the atmosphere. Moreover, an apt prediction of atmospheric temperature is well suited in various cartography studies including Geographic Information Systems (GIS), spatial planning and others, see [8] in connection to cartographic modellings. Traditionally, it falls in the branch of geography which highlights the role of cartography as a map making process. In nutshell, our perspective of cartography encompasses the knowledge in the realism of atmospheric science, philosophy of nature and various techniques incorporated to give a clear picture of spatial planning.

From the outset of this paper, it is worth mentioning that the remote sensing emerges amongst viable techniques being used for studying the characteristics or phenomena undermining an object without any contact with the specimen. Hereby, the remote sensing technology utilizes a suitable sensor in order to detect and classify various objects on the surface of the earth. This mainly works on the propagation of component signals. The source of the foregoing signals can be either from the sun or satellites. In this regard, the weather satellites that are used in climatology emerge among the best applications of remote sensing. Hitherto, the GIS refers to an information system that performs an action of the integration, storing, analyzing, managing and displaying of a particular geographical data. Given the longitude, latitude and altitude in a chosen geographic area, the GIS can correlate various unrelated information [8], as well. The data representation and associated analysis with respect to real objects like the amount of rainfall at a chosen 
location can indeed be examined with an aid of the GIS. Such issues we leave open for a future research exploration.

Following the above mentioned motivations, we examine polynomial based interpolation and extrapolation techniques of the atmospheric temperature in reference to the global warming. Thus, it is worth emphasizing that the interpolation analysis of a given function is used to predict data within a chosen range. However, the extrapolation techniques are used to predict data beyond the range in order for finding an apt behavior of a chosen phenomenon.

\section{MODELLING OF THE ATMOSPHERIC TEMPERATURE}

In this section, we concentrate on possible modeling of the temperature governing the episodes of the El Niño and La Niña which we equally term as the heat and cold waves. Their joint effects are investigated in sequel in Section 3.4 under the agenda of the SOI fine tuning relating large scale interactions between the ocean atmosphere and its climate.

\subsection{FORMULATION OF THE PROBLEM}

In order to model the atmospheric temperature, we are interested in investigating the root cause of the heat and cold waves undermining the global warming. This question requires step by step explanations of the El Niño and La Niña episodes whose joint effects follow from the perspective of SOI, see Section 3.4 below. The associated problem concerning modelling of the atmospheric temperature under flexible expansion of the wind velocity and humidity is formulated as follows:

Let $w$ be the wind velocity, $h$ be the humidity and $T$ be the temperature at a given geographic location in the atmosphere. Thence, for a given set of data $\left\{\left(T_{i}, w_{i}, h_{i}\right) \mid i=\right.$ $0,1,2, \ldots, N\}$ as possible values of the atmospheric parameters $(T, w, h)$, we wish to find the Lagrange polynomial representation of the temperature $T$ as an interpolating function of $(w, h)$. This enables us to determine an approximate behavior of $T(w, h)$ under simultaneous fluctuations of $\{w, h\}$ of the atmosphere.

\subsection{ANALYSIS OF THE TEMPERATURE}

As per the above setup, given the wind velocity $w$, humidity $h$ and the temperature $T$ as a function of $(w, h)$, it is well known [9] that we can express $T(w, h)$ around a given point $\left(w_{0}, h_{0}\right)$ by using its following Taylor series expansion

$$
\begin{gathered}
T(w, h)=T\left(w_{0}, h_{0}\right)+\left.\frac{\partial T}{\partial w}\right|_{\left(w_{0}, h_{0}\right)}\left(w-w_{0}\right)+\left.\frac{\partial T}{\partial h}\right|_{\left(w_{0}, h_{0}\right)}\left(h-h_{0}\right)+\left.\frac{1}{2} \frac{\partial^{2} T}{\partial w^{2}}\right|_{\left(w_{0}, h_{0}\right)}(w- \\
\left.w_{0}\right)^{2}+\left.\frac{1}{2} \frac{\partial^{2} T}{\partial h^{2}}\right|_{\left(w_{0}, h_{0}\right)}\left(h-h_{0}\right)^{2}+\left.\frac{\partial^{2} T}{\partial w \partial h}\right|_{\left(w_{0}, h_{0}\right)}\left(w-w_{0}\right)\left(h-h_{0}\right)+\cdots
\end{gathered}
$$

In the linear case of the expansion of $T(w, h)$ as depicted above in Eqn. (1) with the initial condition $\left(w_{0}, h_{0}\right)=(0,0)$, it follows that we have the following temperature

$$
T(w, h) \simeq T(0,0)+\left.\frac{\partial T}{\partial w}\right|_{(0,0)} w+\left.\frac{\partial T}{\partial h}\right|_{(0,0)} h
$$

Hereby, whenever the humidity $h$ is fixed, it follows that we have the following temperature change $\delta T(w, h)=T_{w}(0,0) w$, where $T_{w}(0,0)$ is the first partial derivative of $T(w, h)$ with respect to $w$ at the point of observation $(0,0)$, viz. we have $T_{w}(0,0)=$ $\left.\frac{\partial T}{\partial w}\right|_{(0,0)}$. On the other hand, it is direct to see that the foregoing change in temperature $T(w, h)$ reads as $\delta T(w, h)=T_{h}(0,0) h$, whenever the wind parameter $w$ is fixed. 
From the standard single variable Taylor series expansion of $T_{1}(w)$, it follows that we have

$$
T_{1}(w)=T_{10} l_{0}(w)+T_{11} l_{1}(w)+T_{12} l_{2}(w)+\cdots
$$

Here, the constants $\left\{T_{1 j} \mid j=0,1,2, \ldots N\right\}$ as in Eqn. (3) represent the Taylor coefficients of $T_{1}(w)$, viz. its $j^{\text {th }}$ order derivative and the functions $\left\{l_{i}(w) \mid \mathrm{i}=1,2, \ldots, \mathrm{N}\right\}$ form the corresponding basis set of the expansion as the Lagrange coefficients, see [10,11]. Hereby, it is observed that the basis functions $l_{i}(w)$ are respectively determined as per the product

$$
l_{i}(w)=\prod_{\substack{j=0 \\ i \neq j}}^{N} \frac{w-w_{j}}{w_{i}-w_{j}}
$$

where $\mathrm{N}$ is the number of interpolation points. In this case, by setting the initial wind velocity $w_{0}=0$ and $N=2$, it is not difficult to see that we have the following set of basis functions

$$
\begin{array}{r}
l_{0}(w)=\left(\frac{w-w_{1}}{w_{0}-w_{1}}\right)\left(\frac{w-w_{2}}{w_{0}-w_{2}}\right)=\frac{w^{2}-w w_{2}-w w_{1}+w_{1} w_{2}}{w_{1} w_{2}} \\
l_{1}(w)=\left(\frac{w-w_{0}}{w_{1}-w_{0}}\right)\left(\frac{w-w_{2}}{w_{1}-w_{2}}\right)=\frac{w^{2}-w w_{2}}{w_{1}^{2}-w_{1} w_{2}} \\
l_{2}(w)=\left(\frac{w-w_{0}}{w_{2}-w_{0}}\right)\left(\frac{w-w_{1}}{w_{2}-w_{1}}\right)=\frac{w^{2}-w w_{1}}{w_{2}^{2}-w_{1} w_{2}}
\end{array}
$$

Herewith, the Table (1) below enables us in considering an interpolation analysis of $T_{1}(w)$ as

\begin{tabular}{|c|c|c|c|}
\hline SN & \# of Counts & $w$ & $T_{1}(w)$ \\
\hline 1 & 0 & $w_{0}=0$ & $t_{0}$ \\
\hline 2 & 1 & $w_{1}$ & $t_{1}$ \\
\hline 3 & 2 & $w_{2}$ & $t_{2}$ \\
\hline 4 & 3 & $w_{3}$ & $t_{3}$ \\
\hline. &. &. &. \\
\hline. &. &. &. \\
\hline
\end{tabular}

Table 1: Observations of the temperature $T_{1}(w)$ as a function of the wind velocity $w$

In this case, by substituting the values of the basis functions $\left\{l_{1}(w), l_{2}(w), l_{3}(w)\right\}$ from Eqns. (4-7) and that of the $\left\{T_{11}, T_{12}, T_{13}\right\}$ as in Table (1) into the Eqn. (3), we find that the corresponding Lagrange polynomial as the wind velocity dependent temperature $T_{1}(w)$ simplifies as

$$
T_{1}(w)=t_{0} \frac{w^{2}-w w_{2}-w w_{1}+w_{1} w_{2}}{w_{1} w_{2}}+t_{1} \frac{w^{2}-w w_{2}}{w_{1}^{2}-w_{1} w_{2}}+t_{2} \frac{w^{2}-w w_{1}}{w_{2}^{2}-w_{1} w_{2}}
$$

On the other hand, when $w$ is fixed, we have $T(w, h)=T_{2}(h)$. In this case, the Lagrange polynomial of the corresponding humidity dependent temperature function $T_{2}(h)$ reads as

$$
T_{2}(h)=\sum_{i=0}^{N} T_{2 i} \tilde{l}_{i}(h)
$$

where the constants $\left\{T_{2 j} \mid j=0,1,2, \ldots, N\right\}$ as in Eqn. (9) represent the Taylor coefficients of $T_{2}(h)$, namely, as the $j^{\text {th }}$ order derivative of $T_{2}(h)$ with respect to the humidity $h$. The basis functions $\left\{\tilde{l}_{i}(h) \mid \mathrm{i}=1,2, \ldots, \mathrm{N}\right\}$ of the expansion are obtained from the corresponding Lagrange coefficients $[10,11]$, which in parallel to Eqn. (4) respectively arise as the product

$$
\tilde{l}_{i}(h)=\prod_{\substack{j=0 \\ i \neq j}}^{N} \frac{h-h_{j}}{h_{i}-h_{j}}
$$


The resulting observations of the humidity function $T_{2}(h)$ are shown in Table (2) as below

\begin{tabular}{|c|c|c|c|}
\hline SN & \# of Counts & $h$ & $T_{2}(h)$ \\
\hline 1 & 0 & $h_{0}=0$ & $t_{0}^{\prime}$ \\
\hline 2 & 1 & $h_{1}$ & $t_{1}^{\prime}$ \\
\hline 3 & 2 & $h_{2}$ & $t_{2}^{\prime}$ \\
\hline 4 & 3 & $h_{3}$ & $t_{3}^{\prime}$ \\
\hline. &. &. &. \\
\hline. &. &. &. \\
\hline
\end{tabular}

Table 2: Observations of the temperature $T_{2}(h)$ as a function of the humidity $h$

Herewith, by following Eqn. (8) with the choice of $h_{0}=0$ and $N=2$, it follows that we have

$$
T_{2}(h)=t_{0}^{\prime} \frac{h^{2}-h h_{2}-h h_{1}+h_{1} h_{2}}{h_{1} h_{2}}+t_{1}^{\prime} \frac{h^{2}-h h_{2}}{h_{1}^{2}-h_{1} h_{2}}+t_{2}^{\prime} \frac{h^{2}-h h_{1}}{h_{2}^{2}-h_{1} h_{2}}
$$

In the limit of small $(w, h)$, we see from Eqn. $(2)$ that the temperature $T(w, h)$ can be estimated as

$$
T(w, h) \simeq T(0,0)+T_{1} w+T_{2} h
$$

With the above expansions of $T(w, h)$ as in Eqn. (12) and component temperature profiles $\left\{T_{1}, T_{2}\right\}$ as depicted in Eqns. $(8,11)$, we find the following effective atmospheric temperature

$$
\begin{gathered}
T(w, h)=C+\left(t_{0} \frac{w^{2}-w w_{2}-w w_{1}+w_{1} w_{2}}{w_{1} w_{2}}+t_{1} \frac{w^{2}-w w_{2}}{w_{1}^{2}-w_{1} w_{2}}+t_{2} \frac{w^{2}-w w_{1}}{w_{2}^{2}-w_{1} w_{2}}\right) w+ \\
\left(t_{0}^{\prime} \frac{h^{2}-h h_{2}-h h_{1}+h_{1} h_{2}}{h_{1} h_{2}}+t_{1}^{\prime} \frac{h^{2}-h h_{2}}{h_{1}^{2}-h_{1} h_{2}}+t_{2}^{\prime} \frac{h^{2}-h h_{1}}{h_{2}^{2}-h_{1} h_{2}}\right) h
\end{gathered}
$$

under variations of the fixed point $\left(w_{0}, h_{0}\right)$ in the proximity of the origin $(0,0)$. In Eqn. (13), $C$ is a constant corresponding to the initial atmospheric temperature $C=T(0,0)$. The cases of large $\{w, h\}$ temperature fluctuations correspond to turbulent El Niño and La Niña episodes, which we leave open for a future research investigation.

\subsection{ERROR ESTIMATION}

In the sequel, we offer permissible errors in an estimation of the above temperature $T(w, h)$ as depicted in Eqn. (13). Namely, for a given wind dependent temperature profile $T_{1}(w)$ as depicted in Eqn. (8), the remainder term $T_{1}{ }^{n+1}(t)\left(w-w_{n}\right) /(n+1)$ ! with $t \in\left[w, w_{n}\right]$ yields the error in its polynomial representation. Refs. $[10,11]$ offer an introduction to the polynomial expansions and associated remainder terms for a given real valued function.

Herewith, for the case of $N=2$, it follows from Eqn. (8) that $T_{1}(w)$ can be simplified as

$$
T_{1}(w)=t_{0} \frac{w^{2}-w w_{2}-w w_{1}+w_{1} w_{2}}{w_{1} w_{2}}+t_{1} \frac{w^{2}-w w_{2}}{w_{1}^{2}-w_{1} w_{2}}+t_{2} \frac{w^{2}-w w_{1}}{w_{2}^{2}-w_{1} w_{2}}+\frac{T_{1}^{N+1}(t)\left(w-w_{n}\right)}{(N+1) !}
$$


For $N=2$, the corresponding error term reads as $T_{1}{ }^{3}(w)\left(w-w_{2}\right) / 3$ !, where $T_{1}{ }^{3}(t)$ is the third order partial derivative of the temperature profile $T_{1}(t)$ with respect to the variable $t$.

On the other hand, given the above effective humidity dependent temperature $T_{2}(h)$ as depicted in Eqn. (11), the associated remainder term $T_{2}{ }^{n+1}(t)\left(h-h_{n}\right) /(n+1)$ ! with $t \in\left[h, h_{n}\right]$ is termed as the error term for its polynomial representation. Thus, with the above remainder term and Eqn. (11), the effective humidity dependent temperature profile $T_{2}(h)$ simplifies as

$$
T_{2}(h)=t_{0}^{\prime} \frac{h^{2}-h h_{2}-h h_{1}+h_{1} h_{2}}{h_{1} h_{2}}+t_{1}^{\prime} \frac{h^{2}-h h_{2}}{h_{1}^{2}-h_{1} h_{2}}+t_{2}^{\prime} \frac{h^{2}-h h_{1}}{h_{2}^{2}-h_{1} h_{2}}+\frac{T_{2}^{N+1}(t)\left(h-h_{N}\right)}{(N+1) !}
$$

Hence, the total error $\Delta T$ in estimating the temperature $T(w, h)$ as above in Eqn. (13) reads as

$$
\Delta T=\max _{n}\left|\frac{T^{n+1}\left(t_{1}\right)\left(w-w_{n}\right)}{(n+1) !}+\frac{T^{n+1}\left(t_{2}\right)\left(h-h_{n}\right)}{(n+1) !}\right|,
$$

where the variables $\left(t_{1}, t_{2}\right)$ respectively represent the wind velocity and humidity pair existing in the atmosphere in their respective intervals of fluctuations $t_{1} \in\left[w, w_{n}\right]$ and $t_{2} \in\left[h, h_{n}\right]$.

\subsection{SOUTHERN OSCILLATION INDEX FINE TUNING}

From the perspective of the above analysis, a joint effect of the El Niño and La Niña episodes is studied via the SOI index in the light of global warming. Namely, the El Niño and La Niña events correspond to the natural global warming and cooling. They occur when the Pacific Ocean and the atmosphere above it changes from the normal states. In this concern, the El Niño refers to large scale ocean atmosphere climate interactions linked to a periodic warming in sea surface temperatures across the central and east central Equatorial Pacific. Further, the El Niño effects change the atmospheric pressure, resulting to rain fall, wind pattern and a rise in the sea surface temperatures, wherefore an increase in the average earth temperature. This is the root cause of the global warming of the earth.

On the other hand, the La Niña effects correspond to periods of decreasing earth temperatures in comparison to its normal state. During the La Niña episodes, places are rather colder and there is a large possibility of having much rain falls and sometimes hurricanes, as well. As far as weather forecasting is concerned, it is worth stating that the La Niña effects are directly opposite to that of the El Niño. However, it is unfortunate that the El Niño effects last longer than the corresponding La Niña counterparts, whereby there would most likely be an overall increase in the earth's temperature.

Finally, the SOI is a standard based on the observed sea level pressure difference between the Tahiti minus Darwin SOI, Australia, see [12] for potential data precisions. In short, the SOI measures large scale fluctuations in air pressure undermining the western and eastern tropical Pacific which respectively occur during the El Niño and La Niña episodes. This is the reason that the El Niño survives for a longer period than the La Niña. An extensive mathematical modeling of the above mentioned atmospheric phenomena we leave open for a future research. 


\section{CONCLUSION}

We provide an interpolating model of the atmospheric temperature under variations of the wind patterns and humidity. Namely, we have successfully obtained an interpolating temperature in the basis of Lagrange polynomials. This is well suited in designing an apt thermometer based on the wind velocity and humidity present in the atmosphere. This analysis is performed under moving fixed point in the vicinity of a chosen observation center. In this paper, we equally estimate the modeling errors in predicting the temperature over simultaneous fluctuations of atmospheric parameters (wind velocity and precipitation). This offers an accurate measurement of the oceanic temperature up to a desired order of precision under different climatic conditions.

In this concern, the El Niño and La Niña episodes emerge as complex weather patterns resulting from different variations in ocean temperatures in the Equatorial Pacific. Namely, our analysis is based on the fact that the El Niño and La Niña effects arise as the opposite phases of the same phenomenon, viz. the ENSO cycles [6-8]. It is worth emphasizing that the ENSO cycle is a scientific measure that describes fluctuations of the atmosphere temperature between the ocean and atmosphere of the east-central Equatorial Pacific. La Niña is hereby referred as the cold phase of the ENSO cycles, while the El Niño as that of the warm phase. Such deviations in the surface temperatures from the corresponding normal states may have adverse impacts on various oceanic processes and the weather, whereby a large scale variation in the climate.

Notice that the El Niño and La Niña episodes typically last 9 to 12 months, but some prolonged events may last for years while their frequency of happening can be equally quite irregular, as well. Typically, the El Niño occurs more frequently than the La Niña, whereby we anticipate an intrinsic relationship between their happenings, affecting both the weather and health. Investigations relating forecasting of the ENSO states and Darwin pressure [12], we prolong for a future research development.

\section{REFERENCES}

[1] IPCC. Climate change 2007: Synthesis report. Contribution of working groups I, II and III to the fourth assessment report of the intergovernmental panel on climate change. 2007. http://www.ipcc.ch/publications_and_data/ar4/syr/ en/contents.html. Accessed March 6, 2016.

[2] Environmental Protection Agency. Climate change. 2010. Available at: www.epa. gov/climatechange. Accessed February 9, 2016.

[3] Ziegler, Carol, Vincent Morelli, and Omotayo Fawibe, Climate Change and Underserved Communities, Primary Care: Clinics in Office Practice 44, no. 1, 171-184, 2017.

[4] Timmermann, Axel, et al., Increased El Niño frequency in a climate model forced by future greenhouse warming, Nature 398, 6729, 694-697, 1999.

[5] E. H. Fouberg, A. B. Murphy, H. J. de Blij, Human Geography: People, Place, and Culture, $11^{\text {th }}$ Edition, Wiley, ISBN-13: 978-1118793145, 2015.

[6] Freidenreich, Stuart M., et al. The new GFDL global atmosphere and land model AM2-LM2: Evaluation with prescribed SST simulations, Journal of Climate 17, 46414673, 2004.

[7] Johnson, Nicholas L, Protecting the GEO environment: policies and practices, Space Policy 15.3, 127-135, 1999. 
[8] Tomlin, Dana C., Geographic information systems and cartographic modeling, Prentice Hall, Englewood Cliffs, NJ, 1990.

[9] Mathews, Jon, and Robert Lee Walker, Mathematical methods of physics, Vol. 501, New York, WA Benjamin, 1970.

[10] R. Courant and D. Hilbert, Methods of mathematical physics, Vol. 1, CUP Archive, 1965 .

[11] Scott, L. Ridgway and Shangyou Zhang, Finite element interpolation of nonsmooth functions satisfying boundary conditions, Mathematics of Computation 54, 190, 483-493, 1990.

[12] Allan, Robert J., et al., A further extension of the Tahiti-Darwin SOI, early ENSO events and Darwin pressure, Journal of Climate 4.7, 743-749, 1991. 